Research Article

Jing Zhang, Pinghua Pan*, Ping Jiang, Jie Qin, and Jiansong Hu

\title{
Electric degradation in PZT piezoelectric ceramics under a DC bias
}

https://doi.org/10.1515/secm-2020-0049

Received Jun 09, 2020; accepted Oct 13, 2020

\begin{abstract}
In order to accurately evaluate the service life and failure mechanism of the PZT piezoelectric ceramics, the electric degradation process of the PZT ceramics with and sans doping under a DC voltage of $380 \mathrm{~V}$, in a surrounding environment of $90^{\circ} \mathrm{C}$ and $85 \% \mathrm{RH}$ has been investigated using a self-made device. The experimental results show that the degradation rate of the pure PZT ceramic is lower than that of ceramics with doping in the same condition. Furthermore, the electrical properties of the ceramics tend to decrease during the electric degradation. The doping increases the defects of ceramics, resulting in that the silver ion transfer from the anode to the cathode under the continuous DC bias, which can further form a metal band, increasing the conductivity, but deteriorating the service life.
\end{abstract}

Keywords: PZT; piezoelectric ceramic; doping; electric degradation; Ag-ion migration

\section{Introduction}

The Lead-zirconate-titanate (PZT) based piezoelectric ceramics are widely applied in various electronic devices because of their excellent electrical properties and low price [1]. Obviously, the pure PZT ceramics possess a relatively poor piezoelectric property, which cannot meet the industrial demand [2]. Hence, the ion doping, a most effectively approach on property-improvements, can increase the amounts of defects, which further achieve the purpose of PZT-modifications [3, 4]. According to the valence states

\footnotetext{
*Corresponding Author: Pinghua Pan: Xinyun Electronic Components Co., Ltd. of China Zhenhua Group, Guiyang, 550018; Hongyun Electronic Components Co., Ltd. of China Zhenhua Group, Guiyang, 550018; Email: phpan083@163.com Jing Zhang: Xinyun Electronic Components Co., Ltd. of China Zhenhua Group, Guiyang, 550018; Hongyun Electronic Components Co., Ltd. of China Zhenhua Group, Guiyang, 550018

Ping Jiang, Jie Qin, Jiansong Hu: Xinyun Electronic Components Co., Ltd. of China Zhenhua Group, Guiyang, 550018
}

O Open Access. (c) 2020 J. Zhang et al., published by De Gruyter. (Cc) BY License and the site of substitutional atoms of the doped ions, it can be divided into two groups as follows, A-site high-valencedoping and B-site low-valence-doping [5, 6]. Nowadays, the majority of research works focus on the composition design to achieve the excellent electrical performances [7, 8]. With the rapid development of electronic information industry, the electronic ceramics are widely applied in extreme environments. Therefore, it is also very important to study the evolutions of microstructure and electrical properties of piezoelectric ceramics in service.

Normally, the long-time service process of the piezoelectrical ceramics is accompanied with the increase of the leakage current, eventually resulting in the breakdown [9]. The above phenomenon is also called as the electric degradation. The relevant researches prove that the high electric field strength, harsh environment (high temperature and humidity, etc.) can exacerbate the service life. Therefore, developing a completely new equipment platform to estimate the service life to deeply understand the failure mechanism has a profound promotion on the commercial applications of the piezoelectric components.

Generally, the service life and failure mechanism of the piezoelectrical ceramics are always lack of careful considerations. In this paper, the PZT ceramics with $\mathrm{Nb}^{5+}$ and $\mathrm{Sb}^{3+}$ doping has been prepared using a traditional solid reaction. And then their electric degradation process under a DC voltage of $380 \mathrm{~V}$, in a surrounding of $90^{\circ} \mathrm{C}$ and $85 \% \mathrm{RH}$ has been investigated using a self-made device through collecting and analyzing the leakage currents. Subsequently, the cross-section morphology was carefully characterized. Also, the variation of the dielectric loss $(\tan \delta)$ and capacitance $\left(C_{p}\right)$ are measured. Finally, the failure mechanism is discussed in detail.

\section{Experimental procedures}

\subsection{Ceramic preparation}

The five metal powders of $\mathrm{Pb}_{3} \mathrm{O}_{4}, \mathrm{ZrO}_{2}, \mathrm{Nb}_{2} \mathrm{O}_{5}, \mathrm{Sb}_{2} \mathrm{O}_{3}, \mathrm{TiO}_{2}$ with the purity over $99.0 \mathrm{wt} . \%$ were chosen as the starting materials. The powders with the nominal compositions

This work is licensed under the Creative Commons Attribution 4.0 
of $\mathrm{Pb}\left(\mathrm{Zr}_{52} \mathrm{Ti}_{48}\right) \mathrm{O}_{3}$ (marked as $\left.1 \#\right), \mathrm{Pb}\left(\mathrm{Zr}_{52} \mathrm{Ti}_{48}\right) \mathrm{O}_{3}-0.1$ wt.\% $\mathrm{Nb}_{2} \mathrm{O}_{5}$ (marked as 2\#) and $\mathrm{Pb}\left(\mathrm{Zr}_{52} \mathrm{Ti}_{48}\right) \mathrm{O}_{3}-0.1$ wt. $\% \mathrm{Sb}_{2} \mathrm{O}_{3}$ (marked as 3\#) were weighed using a AL204 balance, and then blended with alcohol for 24h using a QM-3PS2 ballmill. After dried and sifted, the mixed powders were presintered in $900^{\circ} \mathrm{C}$ for $2 \mathrm{~h}$. After blended and dried again, the powders with $3 \mathrm{wt} . \%$ Polyvinyl alcohol (PVA) were rolled into a round piece with a dimension of $\Phi 20 \times 0.2 \mathrm{~mm}$ using a finishing mill. Subsequently, the ceramic pieces were put into the furnace for sintering at $1200^{\circ} \mathrm{C}$ for $4 \mathrm{~h}$. After the silver electrode coated, the ceramics were polarized by a voltage of $3 \mathrm{kV}$ for $30 \mathrm{~min}$ at $80^{\circ} \mathrm{C}$ in a silicone environment.

\subsection{Electrical degradation test}

The Electrical degradation process was investigated by a self-made device. This device system is composed of six parts as follows: Agilent oscilloscope, $380 \mathrm{~V}$ DC power system, high-low temperature test container, self-made clamp, test circuit and Lab view software. The Schematic diagram of circuit used for electrical degradation is as shown in Figure 1. The experiments were conducted in a high-low temperature test container at $80^{\circ} \mathrm{C}$ and $90 \% \mathrm{RH}$ for 14 days. The leakage current of the ceramics was obtained through collecting the voltage of $R_{1}$ using the oscilloscope.

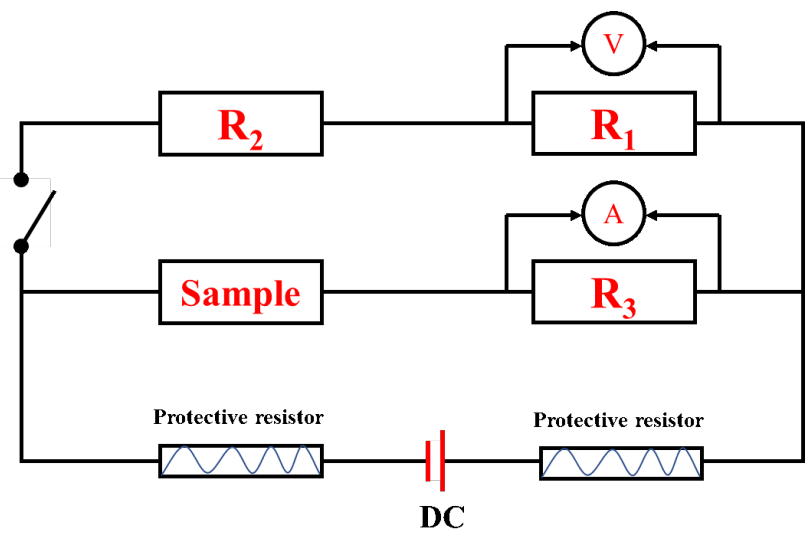

Figure 1: Schematic diagram of circuit used for electrical degradation

\subsection{Ceramic Characterization}

After experiments, the phase structures of ceramics were measured by X'pert powder X-ray diffraction (XRD). A supra40 scanning electron microscope (SEM) was used to observe the cross-section morphologies. A ZJ-3AN quasi-static $d_{33}$ measuring instrument was used to test the $d_{33}$ and $\tan \delta$ of the samples before and after electrical degradations. The capacitance and dielectric loss were measured by TH2618B capacitance tester, and the dielectric constant $\epsilon_{r}$ was calculated according to the dimensions of the ceramics.

\section{Results and discussion}

\subsection{Electrical properties}

Figure 2(a) shows the leakage currents as the function of electric-degradation times. It is seen that the leakage current of the PZT ceramics increase faster than that of pure PZT ceramics. Figure $2 b$ shows the growth rate of the leakage current. Moreover, the ceramics with $\mathrm{Nb}^{5+}$ doping possess the highest increase of leakage current. According to the microcosmic formula (1) of current $j[10]$ :

$$
j=n q s v
$$

where, $n$ is the number of carriers per unit volume, $q$ is the charge number of carriers, $s$ is the cross-sectional area of conductor and $v$ is the migration velocity. It can be suggested that the soft $\mathrm{Nb}^{5+}$ doping can accelerate the electric degradation rate of piezoelectric ceramics because high amounts of defects were formed in the $\mathrm{Nb}^{5+}$-doping ceramics, resulting in the increase of carrier numbers.

Figure 3 shows the variations of $\tan \delta(\mathrm{a})$ and $C_{p}(\mathrm{~b})$ of these three ceramics before and after electric degradation. And their results are displayed in Table 1. After electric degradation, the values of $\tan \delta$ and $C_{p}$ have a little improvement. According to the formula (2):

$$
\tan \delta=\frac{1}{R_{a} \cdot R_{p}}
$$

Where $R_{a}$ is the electrical resistance, $R_{p}$ is the capacitance. The increase of $\tan \delta$ is close reverent with the increase of $R_{a}$ and $R_{p}$. Additionally, the $C_{p}$ can be calculated as follows:

$$
C_{p}=\frac{\epsilon(A}{d}
$$

Where, $\epsilon$ is the permittivity, $A$ is the area of silver electrode, $d$ is the thickness of the ceramics. As is known, the $\epsilon$ is constant value, related to internal properties of materials. The area of silver electrode is also unchangeable during electric degradation. Thus, the increase of $C_{p}$ is attributed to the reduction of effective thickness. 

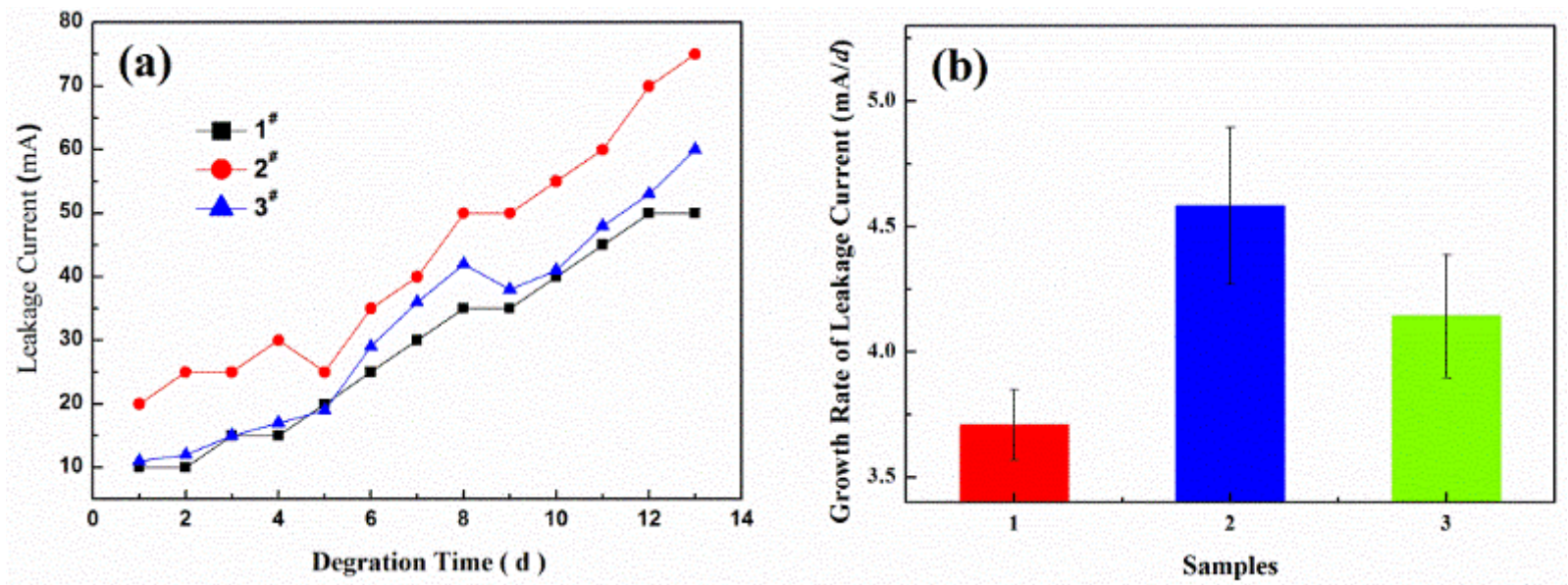

Figure 2: (a) leakage currents as the function of electric-degradation times, (b) growth rate of leakage current
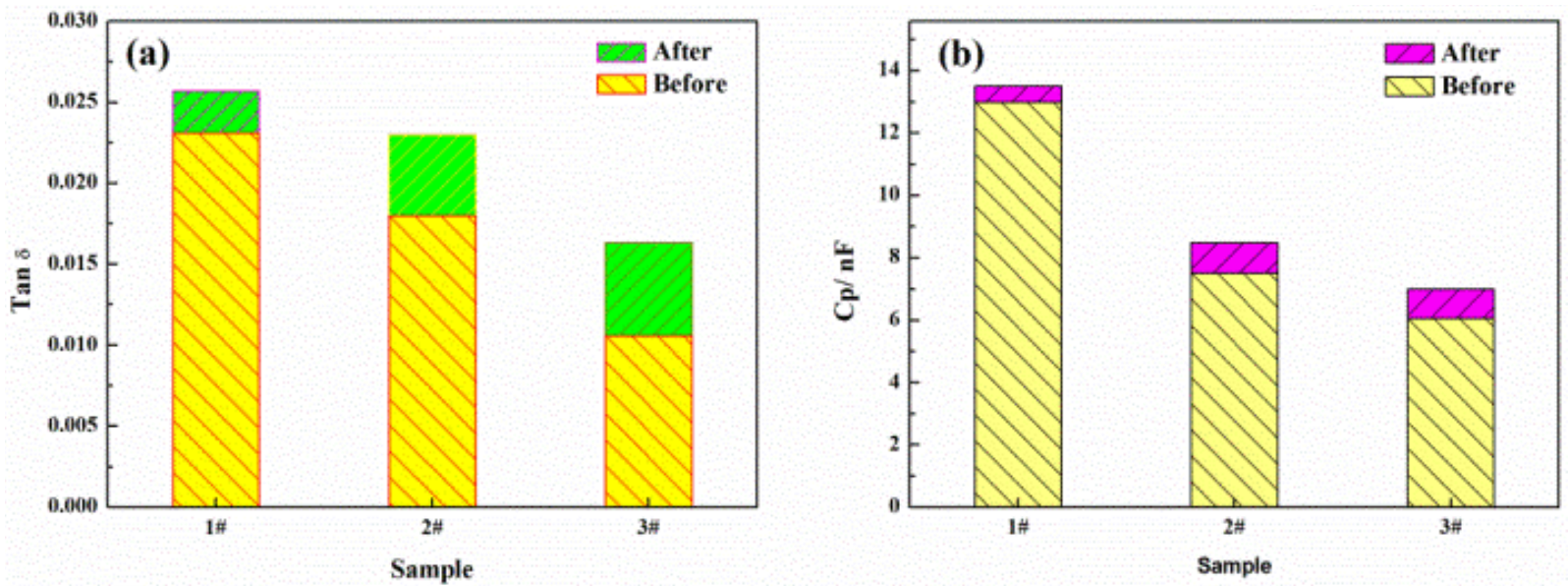

Figure 3: Variations of (a) $C_{p}$ and (b) $\tan \delta$ of these three ceramics before and after electric degradation

Table 1: Value of $C_{p}$ and (b) $\tan \delta$ before and after electric degradation

\begin{tabular}{ccccc}
\hline Samples & \multicolumn{2}{c}{$\tan \delta$} & \multicolumn{2}{c}{$C_{p}$} \\
\cline { 2 - 5 } & Before & After & Before & After \\
\hline $1^{\#}$ & 0.023 & 0.026 & 12.98 & 13.5 \\
$2^{\#}$ & 0.0178 & 0.0228 & 7.45 & 8.31 \\
$3^{\#}$ & 0.010 & 0.0162 & 6.14 & 6.96 \\
\hline
\end{tabular}

\subsection{Phase structures}

Figure 4(a) shows the XRD patterns of the sample 2\# before and after degradation at the $2 \theta$ range from $15^{\circ}$ to $75^{\circ}$. Before electric degradation, the ceramic is composed of a single perovskite structure without any other second phases formed, indicating that $\mathrm{Nb}_{2} \mathrm{O}_{5}$ has been fully dissolved into the perovskite. After electric degradation, the phase structures of the ceramics change a little. However, from the
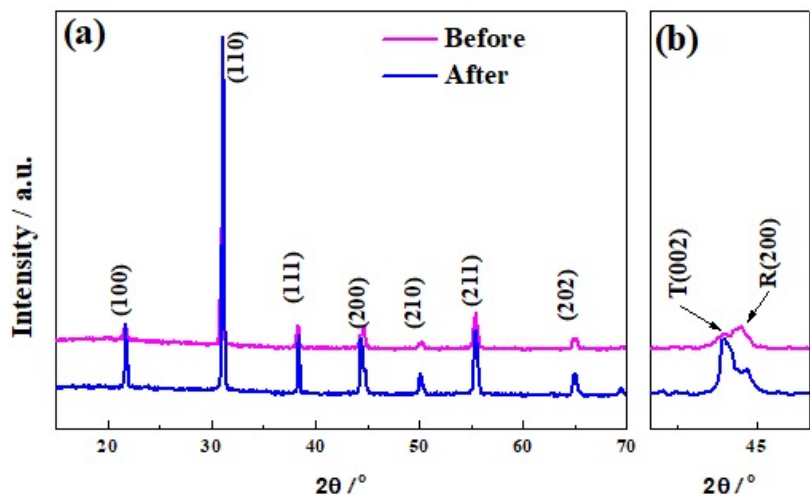

Figure 4: XRD patterns of sample 2\# before and after electric degradation

detail analysis of the (200) and (002) peaks, it can be seen that after electric degradation, the content of tetragonal phases $\mathrm{T}(002)$ increases significantly, which is attributed to that under the action of applied voltage for a long time, 

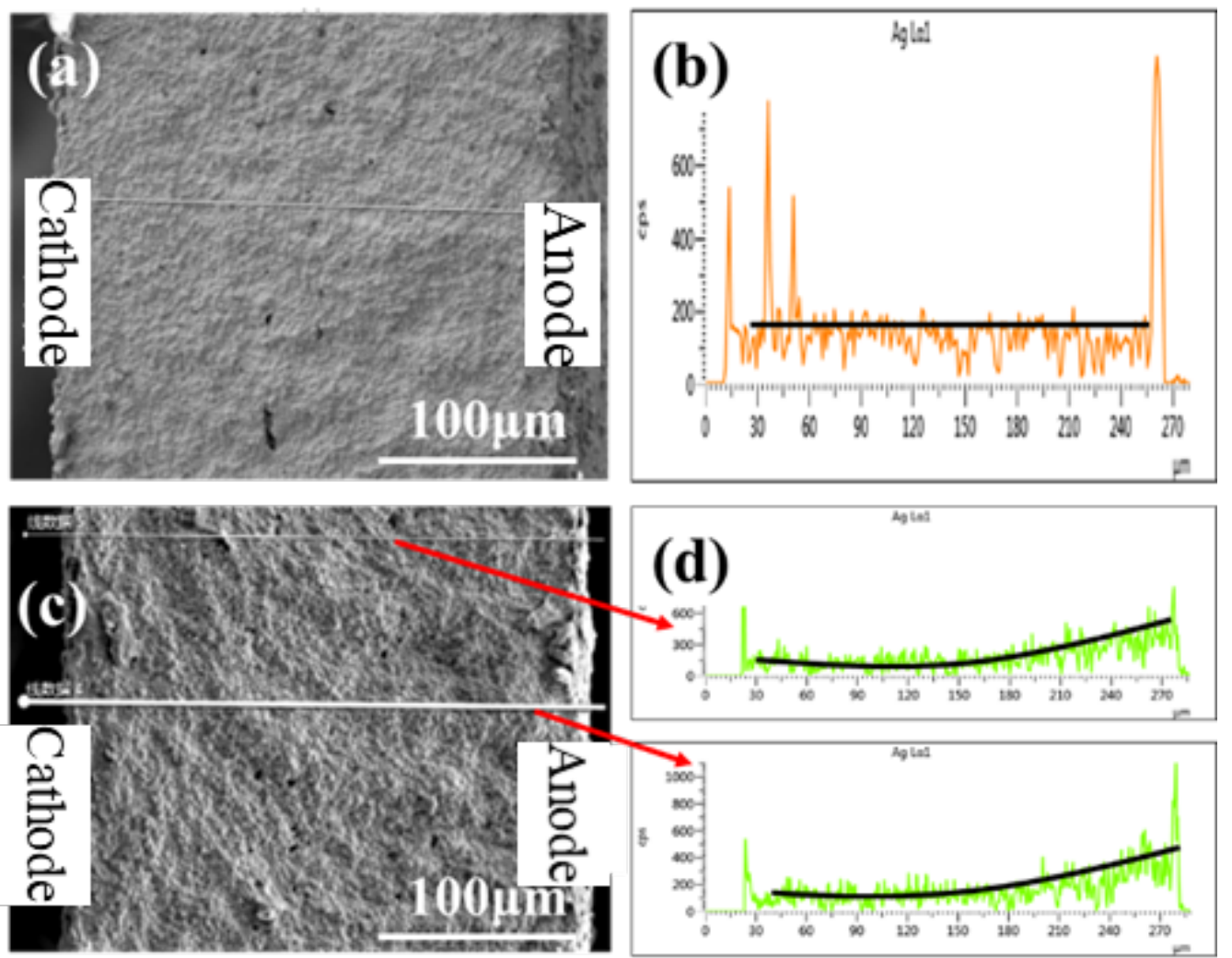

Figure 5: Ag elemental-distribution of sample 2\# (a), (b) before electric degradation; (c), (d) after electric degradation

some electric domains are polarized to translate from a rhombohedral to a rhombohedral phase.

\subsection{Microstructure and Mechanism of electrical degradation}

Figure 5 shows the elemental-distribution of Ag elements in the cross section of the sample 2\#. Before electric degradation, there is no Ag elements detected in the mapping. As for the ceramic after electric degradation, the high content of Ag element appears near the anode, demonstrating that the silver ions on the positive electrode migrate to the negative electrode effected by the external electric field.

Bouyssou believe that the degradation is due to the $\mathrm{Pb}$ ion vacancy and its migration in the process of electrical degradation [11]. Lehovec's degradation model proposed that the electrical degradation is due to the high mobility of oxygen vacancy in undoped and doped samples [12]. These two theories can explain the effect of doping and defect concentration on the electrical degradation of ceramics. Additionally, E. Loh put forward a grain-boundary model that the electric field damaged the internal grain boundaries of ceramics, accelerating the electrical degradation [13]. This model can solve the influence of microstructure on electrical degradation. Moreover, according to these theories and models, Thongrueng considered that under the extreme condition of high temperature and high humidity, some $\mathrm{Ag}$ atoms are ionized. In the electric field, $\mathrm{Ag}^{+}$ion migrates from the positive electrode to the negative electrode. Because of the defect position such as grain boundary, the arrangement of atoms is disordered, the activation energy of $\mathrm{Ag}^{+}$diffusion is small and easy to migrate [14]. Our research works completely coincide with Thongrueng's thoughts. And the schematic diagram of Ag-ion migration during the electric degradation is shown in Figure 6. Mean-

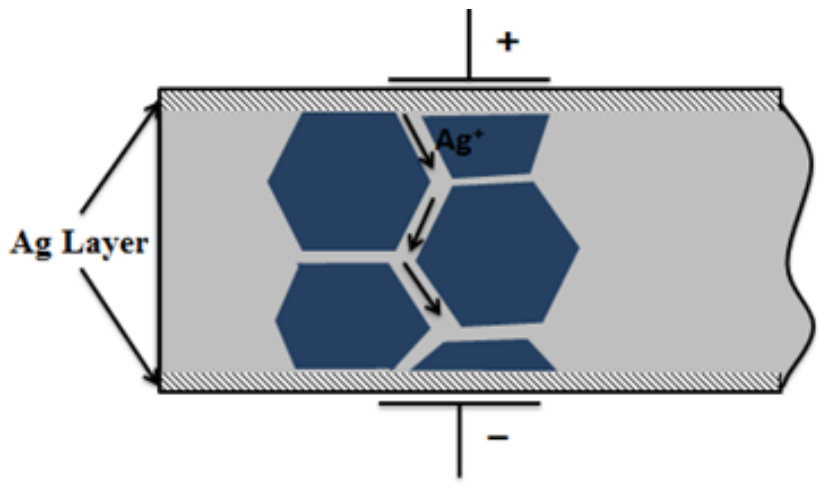

Figure 6: Shows the schematic diagram of Ag-ion migration 
while, amounts of defects, such as vacancy and free electron, are produced in the $2 \#$ piezoelectric ceramics. In the electric field for a long time being, the defects migrate directionally to form the microleakage current. Therefore, the rate of electrical degradation is faster than that of the sample $1^{\#}$ and $3^{\#}$.

\section{Conclusions}

In the condition of $380 \mathrm{~V}$ DC load, $90^{\circ} \mathrm{C}$ and $85 \% \mathrm{RH}$, the electric degradation process of pure and doped PZT was studied. It was found that the electrical degradation rate of undoped PZT samples was slower than that of doped PZT ceramic. Indeed, the doping accelerated the electrical degradation rate of piezoelectric ceramics, promoted the reduction of piezoelectric properties and failure of samples. The two piezoelectric ceramics with ion-doping increases the internal defects. With the increase of working time, Agions move from positive electrode to negative electrode, increasing the conductivity and current density of piezoelectric ceramics. This is the main reason for the degradation process of piezoelectric ceramics. The related results can provide a reference for the evaluation of the service life of piezoelectric ceramics.

CRediT authorship contribution statement: Jing Zhang: Writing - original draft, Data curation. Pinghua Pan: conceptualization, methodology, review \& editing. Ping Jiang: methodology and supervision. Jie Qin: conceptualization, resources. Jiansong Hu: Methodology.

Acknowledgement: The author greatly thanks for the industrial research project of Guizhou provincial science and technology department, China (NO. (2015)3005).

\section{References}

[1] Peng Gui Gui, Zheng De Yi, Cheng Cheng, Zhang Jing, Zhang Hao. Effect of rare-earth addition on morphotropic phase boundary and relaxation behavior of the PNN-PZT ceramics. Journal of Alloys \& Compounds. 2017. 693. 1250-1256
[2] Liu Hong, Nie Rui, Yue Yang, Zhang Qian, Chen Qiang, Zhu Jianguo, Yu Ping, Xiao Dingquan, Wang Changlong, Wang Xingyi. Effect of $\mathrm{MnO}_{2}$ doping on piezoelectric, dielectric and ferroelectric properties of PNN-PZT ceramics. Ceramics International. 2015. 41(9). 11359-11364

[3] Peng Gui Gui, Zheng De Yi, Hu Shun Min, Zhao Hao, Cheng Cheng, Zhang Jing. Effects of rare-earth $\mathrm{Sm}_{2} \mathrm{O}_{3}$ addition on relaxation behavior and electric properties of $0.5 \mathrm{PNN}-0.5 \mathrm{PZT}$ ceramics. Journal of Materials Science Materials in Electronics. 2016. 27(6). 5509-5516

[4] Kim Yong Jin, Yoo Juhyun, Lee Jie Young. Piezoelectric properties of PCW-PNN-PZT ceramics sintered at low temperature. Ferroelectrics Letters. 2017. 44(1-3). 1-7

[5] Liu Qun, Sun Qingchi, Ma Weibing, Li Minmin, Xu Qing, Zhang Qi. Large-strain $0.7 \mathrm{~Pb}\left(\mathrm{Zr}_{x} \mathrm{Ti}_{1-\chi}\right) \mathrm{O}_{3}-0.1 \mathrm{~Pb}\left(\mathrm{Zn}_{1 / 3} \mathrm{Nb}_{2 / 3}\right) \mathrm{O}_{3}$ $0.2 \mathrm{~Pb}\left(\mathrm{Ni}_{1 / 3} \mathrm{Nb}_{2 / 3}\right)_{3}$ piezoelectric ceramics for hightemperature application. Journal of the European Ceramic Society. 2014. 34(5). 1181-1189

[6] Peng Guigui, Chen Chen, Jing Zhang, Zheng Deyi, Hu Shunmin, Hao Zhang. Effects of PNN/PZT ratios on phase structure, electric properties and relaxation behavior of PZN-PNN-PZT ceramics. Journal of Materials Science Materials in Electronics. 2016. 27(4). 3145-3151

[7] Shuai Cheng, Zhang Bo-Ping, Lei Zhao, Wang Kai-Kun. Enhanced insulating and piezoelectric properties of $0.7 \mathrm{BiFeO}_{3}-0.3 \mathrm{BaTiO}_{3}$ lead-free ceramics by optimizing calcination temperature: analysis of $\mathrm{Bi}^{3+}$ volatilization and phase structures. Journal of Materials Chemistry C. 2018. 6

[8] Peng Ze-Hui, Zheng De-Yi, Zhou Tao, Yang Liu, Zhang Ning, Fang Chao. Effects of $\mathrm{CO}_{2} \mathrm{O}_{3}$ doping on electrical properties and dielectric relaxation of PMS-PNN-PZT ceramics. Journal of Materials Science: Materials in Electronics. 2018. 29(7). 5961-5968

[9] Zheng Deyi, Luo Min, Swingler Jonathan. Multi-breakdown model for explaining the formation and growth of black spots in PZT capacitor under DC bias. Sensors and Actuators A: Physical. 2016. 241. 197-202

[10] Ness H., Dash L. K. Many-body current formula and current conservation for non-equilibrium fully interacting nanojunctions. Journal of Physics A 2012. 45(19). 195301

[11] Bouyssou E., Jerisian R., Cezac N. Wafer level reliability and leakage current modeling of PZT capacitors. Materials Science \& Engineering B. 2005. 118. 28-33

[12] Lehovec, Kurt. Space-Charge Layer and Distribution of Lattice Defects at the Surface of Ionic Crystals. Journal of Chemical Physics. 1953. 21(7). 1123

[13] Loh E., Couch F. J., Hendricksen C., Farid L., Weber B. L. Development of donor-derived prostate cancer in a recipient following orthotopic heart transplantation. Jama the Journal of the American Medical Association. 1997. 277(2). 133-137

[14] Thongrueng Jirawat, Tsuchiya Toshio, Nagata Kunihiro. Lifetime and Degradation Mechanism of Multilayer Ceramic Actuator. Japanese Journal of Applied Physics. 1998. 37. 5306-5310 